\title{
A probabilistic and automated tool for the vetting of transit candidates
}

\author{
Olivier Demangeon ${ }^{1}$ and Pascal Bordé ${ }^{2}$ \\ ${ }^{1}$ Aix Marseille Université, CNRS, LAM (Laboratoire d'Astrophysique de Marseille) UMR 7326 \\ 13388, Marseille, France \\ email: olivier.demangeon@lam.fr \\ ${ }^{2}$ Université de Bordeaux, Observatoire Aquitain des Sciences de l'Univers, BP 89, \\ 33271 Floirac Cedex, France \\ e-mail: pascal.borde@u-bordeaux.fr
}

\begin{abstract}
We developed, based on the CoRoT experience, an automated tool called BART for Bayesian Analysis for the Ranking of Transit in order to perform a homogeneous and automated ranking of planetary candidates. We applied it to the candidates detected in the campaign 1 of $\mathrm{K} 2$.
\end{abstract}

Keywords. planets and satellites: general, methods: statistical, techniques: photometric

\section{Introduction: Need for automated vetting of planetary candidates}

CoRoT (Baglin et al. 2006), Kepler (Koch et al. 2010) and now K2 (Howell et al. 2014) have to deal with tens of thousands of transit events. Such events are well known to be treacherous as they can reveal as easily planets and eclipsing binaries. CoRoT, Kepler and K2 planet harvests are thus hiding a tremendous effort in order to vet, follow-up and finally reveal the planetary nature of some of these transits (for eg. Léger et al. 2009). If up to now this process as been mainly done "manually" by vetting and follow-up teams, the future TESS and Plato missions will require automation. Follow-up observations are very difficult to automatize, but the vetting process is much more suitable for that. Recently, automated tools to vet the Kepler Objects of Interest have appeared, see Morton 2012 and Coughlin et al. 2015. We are going to present a tool, developed on the experience acquired with the CoRoT, that we applied to the candidates found in K2 campaign 1.

\section{Method: The bart software}

BART for Bayesian Analysis for the Ranking of Planets is an automated software to rank planetary candidates based on the probability for a given transit to be due to a planet orbiting the target star. This probability is computed in the framework of Bayesian model comparison. The set of models is : planetary system (P.S., a planet orbiting the target star), contaminating planetary system (C.P.S., a planet orbiting another star which contaminates the light curve of the target star), eclipsing binaries (E.B., a star orbiting the target star) and contaminating eclipsing binaries (C.E.B., the eclipsing binary doesn't involve the target star). The models E.B. and C.E.B. are actually divided into two separated models depending on whether the real orbital period of the eclipsing binary is the detected one (E.B.P.) or if it's the double of it (E.B.2.P.) due to similar depths of the primary and secondary eclipses. Finally, it's important to mention that hierarchical triple system model is included in C.P.S. or C.E.B.P., C.E.B.2.P. models depending on the 
Table 1. Fragment of the ranking for K2 campaign 1

\begin{tabular}{cccccccccc}
\hline Rank & Candidate & \multicolumn{2}{c}{ Nature } & \multicolumn{4}{c}{ Probability of the different models [\%] } \\
& & $(\mathrm{M}+15)$ & $($ BART $)$ & P.S. & C.P.S. & E.B.P. & E.B.2.P. & C.E.B.P. & C.E.B.2.P. \\
\hline 1 & 201569483.01 & FP & PS & 99.7 & 0.17 & 0.0 & 0.0 & 0.13 & 0.0 \\
2 & 201779067.01 & FP & PS & 71.6 & 28.4 & 0.0 & 0.0 & 0.0 & 0.0 \\
3 & 201565013.01 & Candidate & PS & 52.9 & 42.9 & 0.15 & 0.0 & 4.0 & 0.05 \\
4 & 201367065.01 & Planet & PS & 51.64 & 47.4 & 0.07 & 0.04 & 0.85 & 0.05 \\
\hline
\end{tabular}

nature of the eclipsing system. For all these models the set of parameters are: orbital period, radius ratio, temperature ratio, impact parameter, density proxy $\rho^{\prime}=\frac{M_{\text {prim }}+M_{s e c}}{4 / 3 \pi R_{\text {prim }}^{3}}$, and the contamination of the light-curve.

All those models are confronted to the detected transits, but contrary to Morton 2012, in order to reduce the computational time, we don't use the folded transit light-curve directly, but a set of observables measured on this light-curve : detected period of the transit, odd and even depths, secondary depth, inner and outer durations.

In order to automatize the computation, the exploration of the parameter space is done thanks to an adaptation of Gregory 2005 Automated Metropolis-Hasting MCMC algorithm. The adaptation consists in including an estimate of the autocorrelation of the trace of each free-parameter into the loop which adapt the size of the proposal distribution. This exploration allows to do parameters inference for each of the models and then to define a narrowed parameter space for the computation of the un-normalized posterior probability of each model thought Monte Carlo Integration. Finally the probability of each model is normalized by the sum of all the considered models. Then all the studied transits are ranked according to the posterior probability of the P.s. model.

\section{Results and discussion}

We applied BART to the candidate list announced in Montet et al. 2015 thereafter $\mathrm{M}+15$. Due to space limits, we only present in table 1 the first 4 candidates. The first two candidates in our ranking are mentioned as false positive $\mathrm{M}+15$. But this is easily explained by our non-detection of the secondary eclipses due to our circular orbit hypothesis. This is a known weakness which will be corrected soon. The third candidate is also still a candidate in $\mathrm{M}+15$ and the fourth turns out to be one of the K2-3 planets Crossfield et al. 2015. Those results are thus very promising. We will thus apply BART to all the candidates detected by K2 in the near future. Such an automated analysis will be of particular interest for the estimate of the reliability and completeness of planet detection with $\mathrm{K} 2$.

\section{References}

Baglin, A., Auvergne, M., Boisnard, L. et al. 2006, 36th COSPAR Scientific Assembly, 36, 3749 Coughlin J. F. et al. 2015, Submitted to ApJ

Gregory 2005, Cambridge University Press

Howell S. B. et al. 2014, PASP, 126, 398H

Koch D. G. et al. 2010, ApJ, 713, 79

Léger A. et al. 2010, A\&A, 506, 287

Montet, B., Morton T. D., Foreman-Mackey, D., et al. 2015, ApJ, 809, 25

Morton, T. D. 2012, ApJ, 761, 6 